Research Paper

\title{
The Effect of Rosmarinic Acid on Neural Differentiation of Wartons Jelly-derived Mesenchymal Stem Cells in Two-dimensional and Three-dimensional Cultures using Chitosan-based Hydrogel
}

\author{
Mohsen Salmanvandi ${ }^{1}$ (D), Seyed Mohammad Amin Haramshahi ${ }^{2,3}$ (D), Elahe Mansouri ${ }^{4}$ (D), Akram Alizadeh A $^{\star}$ (iD) \\ 1. Department of Material Engineering, Najafabad Branch, Islamic Azad University, Najafabad, Iran. \\ 2. Cellular and Molecular Research Center, Iran University of Medical Sciences, Tehran, Iran. \\ 3. Department of Tissue Engineering and Regenerative Medicine, Faculty of Advanced Technologies in Medicine, Iran University of Medical Sciences, \\ Tehran, Iran. \\ 4. Laboratory of Research and Development of Tissue Engineering Products-Hazrat Fatemeh hospital, Iran University of Medical Sciences, Tehran, Iran \\ 5. Department of Tissue Engineering and Applied Cell Sciences, Faculty of Medicine, Semnan University of Medical Sciences, Semnan, Iran
}

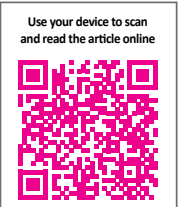

Citation Salmanvandi, M., Haramshahi, S. M. A., Mansouri, E., \& Alizadeh, A. (2023). The Effect of Rosmarini Acid on Neural Differentiation of Wartons Jelly-derived Mesenchymal Stem Cells in Two-dimensional and Threedimensional Cultures using Chitosan-based Hydrogel. Basic and Clinical Neuroscience, 14(1), 117-128. http://dx.doi. org $/ 10.32598 / \mathrm{bcn} .2021 .2596 .1$

http://dx.doi.org/10.32598/ben.2021.2596.1

Article info:

Received: 23 May 2020

First Revision: 15 Aug 2020

Accepted: 25 Aug 2020

Available Online: 01 Jan 2023
Keywords:

Rosmarinic acid, Neural differentiation, Hydrogel, Chitosan $(\mathrm{CH})$, Wharton's jelly-derived mesenchymal stem cells (WJMSCs)

\section{A B S T RA C T}

Introduction: Numerous studies have shown the positive effects of rosmarinic acid on the nervous system.Rosmarinic acid as a herbal compound with anti-inflammatory effects can prevent thedestructive effect of inflammation on the nervous system. Furthermore, various studies haveemphasized the advantages of three-dimensional (3D) culture over the twodimensional (2D) culture of cells.

Methods: In this study, thermosensitive chitosan $(\mathrm{CH})$-based hydrogel as a 3D scaffoldwith the combination of chitosan, beta-glycerol phosphate and hydroxyl ethyl cellulose (CH-GP-HEC) loaded with rosmarinic acid was used to induce neuronal differentiation in humanWharton jelly stem cells. Also, cells were divided into eight groups to evaluate the effect of 3Dcell culture and to compare gene expression in different induction conditions.

Results: The results ofgene expression analysis showed the highest expression of neuronal markers in Whartons jelly derived mesenchymal stem cells (WJMSCs) cultured in chitosan, beta-glycerol phosphate and hydroxyl ethyl cellulose (ch-gp-hec) loaded with differentiation medium androsmarinic acid. According to the results of gene expression, rosmarinic acid alone has a positiveeffect on the induction of expression of neural markers. This positive effect is enhanced by cellculture in 3D conditions.

Conclusion: This study shows that rosmarinic acid can be considered an inexpensiveand available compound for use in neural tissue engineering. The results of this study indicatethat rosmarinic acid can be considered a cheap and available compound for use in neural tissueengineering.

\section{* Corresponding Author:}

Akram Alizadeh, PhD.

Address: Department of Tissue Engineering and Applied Cell Sciences, Faculty of Medicine, Semnan University of Medical Sciences, Semnan, Iran. Tel: +98 (935) 9503052

E-mail:alizadehbio@gmail.com 


\section{Highlights}

- The rosmarinic acid can neuro-differentiates

- Wartons Jelly-Derived Mesenchymal Stem Cells

- 3D chitosan based hydrogel enhances neuro-diffrentiation effect of the rosmarinic acid

- The rosmarinic acid can be considered a cheap and available compound for use in neural tissue engineering

\section{Plain Language Summary}

As rosmarinic acid is a herbal compound with anti-inflammatory effects we used it for differentiation of Whartons Jelly derived mesenchymal stem cells (WJMSCs). Also considering the advantages of three-dimensional (3D) culture, thermosensitive chitosan $(\mathrm{CH})$-based hydrogel as a 3D scaffold loaded with rosmarinic acid was used to induce neuronal differentiation in human Wharton jelly stem cells. The results of gene expression analysis showed the highest expression of neuronal markers in Whartons jelly derived mesenchymal stem cells (WJMSCs) cultured in 3D chitosan based hydrogel loaded with differentiation medium and rosmarinic acid. This study shows that rosmarinic acid can be considered an inexpensive and available compound for use in neural tissue engineering. The results of this study indicate that rosmarinic acid can be considered a cheap and available compound for use in neural tissue engineering.

\section{Introduction}

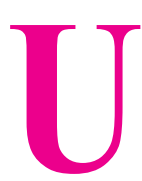

lcerative colitis (UC) inflammation is one of the causes of damage to the central nervous system (CNS). Therefore blocking the molecular pathway of inflammation can enhance nervous system regeneration (Rust \& Kaiser, 2017). Rosmarinic acid is one of the herbal phenolic compounds known for its anti-inflammatory, antioxidant, anti-bacterial, and neuroprotective properties (Adomako-Bonsu et al., 2017; Fonteles et al., 2016; Hase et al., 2019; Nieto et al., 2018). The neuroprotective effects of Retinoic Acid (RA) have been investigated in various diseases, such as Alzheimer's and Parkinson's diseases using animal models (Hamaguchi et al., 2009; Wang et al., 2012). Studies have shown that RA increases the expression of neurotrophic factors, such as brain-derived neurotrophic factor (BDNF), which has an essential role in the plasticity of synapses (Hwang et al., 2016; Lu et al., 2015).

On the other hand, cell-based tissue engineering has been widely considered to repair the central nervous system. various types of mesenchymal stem cells (MSCs) have been studied for this purpose (Simorgh et al., 2019). Wharton's jelly-derived mesenchymal stem cells (WJMSCs) as a critical source of mesenchymal stem cells have higher proliferation and differentiation potential than bone marrow mesenchymal stem cells (Bharti et al., 2018; Wang et al., 2004). They preserve their stem cell properties even after frequent passages (Allahbakhshi et al., 2013). The immune response to these cells is very low and they are not tumorigenic, and due to the role of modulating the immune response from these cells, it can be used in allogeneic transplantation (Vawda \& Fehlings, 2013). Many studies have demonstrated the differentiation of Wharton jelly mesenchymal stem cells to neurons in vitro and in vivo. One study showed that WJMSCs can differentiate into Schwann cells in the presence of glial growth factors. The same study also found the medium condition of differentiated WJMSC can increase the neurite outgrowth in the pheochromocytoma (PC)12 cell line (Peng et al., 2011). Differentiated WJMSC has also been shown to improve neuronal function in the rat brain after ischemic stroke (Zhang et al., 2006).

Hydrogels are similar to soft tissues in terms of water content and mechanical properties. They also have good oxygen permeability and high biocompatibility. Chitosan $(\mathrm{CH})$ is a natural biocompatible and biodegradable polysaccharide obtained by the deacetylation of chitin. $\mathrm{CH}$ can be dissolved in aqueous acidic solutions due to its chemical structure (Supper et al., 2014). It has an anti-free radical and neuroprotective effect and an adjustable degradation rate that support neural cells to adhesion and growth (Gnavi et al., 2013). CH has an anti-bacterial feature (Fregnan et al., 2016) and supports the neural differentiation of stem cells (Zhang et al., 2016). Various studies have been performed on the use of chitosan and its derivatives in tissue engineering, including nervous system tissue engineering. 
In a study, photocrosslinkable chitosan-based hydrogels were used for nerve tissue engineering and it was shown that chitosan-based hydrogels can induce neuronal differentiation in neural stem cells (Valmikinathan et al., 2012). Chitosan has also been widely used to produce thermosensitive hydrogels. Thermosensitive hydrogel scaffolds can be used to transport stem cells and drugs (Liu et al., 2016). They mimic the natural extracellular matrix and provide a suitable environment similar to three-dimensional (3D) tissue for cell growth (Shen et al., 2015; Supper et al., 2014). Crompton et al. showed that polylysine-functionalized thermoresponsive chitosan hydrogel is a suitable scaffold for nerve tissue engineering (Crompton et al., 2007). Studies have shown that $\beta$-glycerol phosphate ( $\beta$ GP) at $37^{\circ} \mathrm{C}$ can act as a catalyst in the sol-gel transfer of chitosan solution (Pankongadisak \& Suwantong, 2018). Furthermore, hydroxyethyl cellulose (HEC) is used as a protective colloid in the polymerization process (Zulkifli et al., 2019). Various studies have used CH-- $\beta$ GP-HEC (to make thermosensitive hydrogels for use in tissue engineering). Due to its low cytotoxicity, this hydrogel can be used as a stem cell carrier for liver tissue engineering (HaddadMashadrizeh et al., 2013). Karimpour Malekshah and colleagues also found that $\mathrm{CH}-\beta \mathrm{GP}-\mathrm{HEC}$ can be used to induce chondrogenic differentiation in adipose tissue-derived mesenchymal stem cells (Karimpour Malekshah et al., 2016).

To investigate the difference between two-dimensional (2D) and 3D cell cultures, various culture systems can be selected. Khodabandeh et al. used 2D collagen films and 3D collagen scaffolds for the culture of HWJMSCs. (Khodabandeh et al., 2016). In another research, Hosseini et al. used 3D alginate scaffolds and a 2D monolayer system to differentiate WJMSCs into neurons (Hosseini et al., 2015). In a study, perinatal MSC from Wharton's Jelly of the umbilical cord (UC-MSC) was used for their differentiation capacities due to partial expression of pluripotency markers in bone tissue engineering. To this end, UC-MSCs were cultured on 3D collagen I/III gels and osteogenic differentiation occurred, showing all features needed for effective bone fracture healing (Schneider et al., 2010). Bagher et al. investigated the effect of the 3D nano-scaffolds on (WJMSCs) differentiation into neuronal motor lineages in the presence of RA and Sonic Hedgehog (SHH). The results showed adhesion, proliferation, and differentiation of WJMSCs (Bagher et al., 2016). In another study, a 3D biomimetic nano-hydroxyapatite/chitosan/gelatin (nHA/CH/Gel) scaffold was used to differentiate WJMSCs. Results indicated that WJMSCs attached to the scaffold surface and uniformly spread throughout the contacting surface (Jamalpoor et al., 2019).
This study was conducted to assay the effect of rosmarinic acid in the neural differentiation of WJMSC in three dimensions culture using a thermosensitive chitosan-based hydrogel (CH-hydrogel).

\section{Materials and Methods}

\section{Study design}

To evaluate the effect of RA-loaded $\mathrm{CH}$-hydrogel on the induction of neural differentiation in WJMSCs, WJMSCs were divided into several groups, including A) WJMSCs (as a control group)

B) WJMSCs+neural differentiation medium

C) WJMSCs+CH-hydrogel loaded with RA

D) WJMSCs+CH-hydrogel loaded with $\mathrm{RA}+$ neural differentiation medium

E) WJMSCs+RA+neural differentiation medium

F) WJMSCs+RA

G) WJMSCs+CH-hydrogel

H) WJMSCs+CH-hydrogel+neural differentiation medium

\section{WJMSCs isolation and characterization}

Umbilical cord samples were collected from full-term newborns from healthy mothers under ethical consent and transferred into a lab in sterile phosphate buffer saline (PBS, Sigma-Aldrich, USA) containing 3\% W/V penicillin (100 IU/mL, Sigma Aldrich, US)/streptomycin $(100 \mu \mathrm{g} / \mathrm{mL}$, Sigma Aldrich, US). Samples were washed twice using sterile PBS and then cut into small parts and vessels were removed. Afterward, Wharton jelly was chopped (into approximately $1 \mathrm{~mm} 3$ pieces) and directly transferred into cell culture flasks containing Dulbecco's modified eagle medium/nutrient mixture F-12 (DMEM/F-12) containing 10\% fetal bovine serum (FBS; Gibco) and incubated at $37^{\circ} \mathrm{C}$ in a carbon dioxide (CO2) incubator. The cell was passaged using $0.25 \%$ W/V trypsin/ Ethylenediaminetetraacetic Acid (EDTA), and cells were used on passage 3 . The isolated cells from passage 3 were characterized using flow cytometry (BD FACS Calibur Bioscience, USA) for CD90, CD44, CD105, and CD74 as mesenchymal stem cells positive markers and CD34, CD45 as negative markers. Accordingly, the cells were incubated with antibodies against CD90 (1:200), CD44 (1:300), CD34 (1:100), and CD45 
(1:100) according to the manufacturer's instructions. The results were analyzed using FlowJo software (version 7.6.1)

\section{Fabrication of chitosan-hydrogel}

A total of $225 \mathrm{mg} \mathrm{CH}$ (Sigma Aldrich-448869-50g) was dissolved in $9 \mathrm{~mL}$ of $0.1 \mathrm{M}$ hydrochloric acid by mechanical shaking. The prepared solution was autoclaved and after cooling, it was kept at $4^{\circ} \mathrm{C}$ until use. Next, cold $\beta$ GP solution $(3 \% \mathrm{~W} / \mathrm{V}$ in deionized water sterilized via filtration using a $0.22 \mu \mathrm{m}$ syringe filter) was added dropwise to the cold $\mathrm{CH}$ solution with continuous stirring. After adding $\beta G P$, the final volume of the solution reached $15 \mathrm{~mL}$. For the preparation of the HEC solution, $125 \mathrm{mg}$ of HEC (Sigma Aldrich) was dissolved in 10 $\mathrm{mL}$ of PBS. HEC solution and $\mathrm{CH}-\beta-\mathrm{GP}$ solution were mixed with a ratio of 1:6 To prevent gelling formation, $\mathrm{CH}-\beta-\mathrm{GP}$ was stored on ice and the HEC solution was added to $\mathrm{CH}-\beta-\mathrm{GP}$ just before injection. Separately to prepare RA stoke, $10 \mathrm{mg}$ of RA Sigma (R4033-10MG) was dissolved in $500 \mu \mathrm{L}$ DMSO. RA and cells were also added to $\mathrm{CH}-\beta \mathrm{GP}-\mathrm{HEC}$ before gelation.

\section{Scanning electron microscopy (SEM)}

The hydrogel morphology and porosity were investigated using scanning electron microscopy (SEM) (HITACHI-S4160). To complete the gelation process, the RA was added toCH- $\beta$ GP-HEC solution, and the final solution was transferred to $37^{\circ} \mathrm{C}$ for $1 \mathrm{~h}$. Before freezedrying, samples were kept at $-80^{\circ} \mathrm{C}$. samples were gold coated using a sputter coater (Technics, Hummer II, Japan) before SEM.

\section{Cytotoxicity assay}

A 2,5 diphenyl tetrazolium bromide (MTT) assay was performed to evaluate cytotoxicity three days after seeding cells on the hydrogel. $5 \times 10^{3}$ WJMSCs per $\mathrm{cm}^{2}$ were seeded in a 96-well plate containing prepared rosmarinic acid-loaded hydrogels and culture media then incubated for $72 \mathrm{~h}$. After removing the cell culture medium, 200 $\mu \mathrm{L}$ MTT solution $(0.5 \mathrm{mg} / \mathrm{mL}$ in PBS) was added into each well, and cells were incubated at $37^{\circ} \mathrm{C}$ for $4 \mathrm{~h}$. Afterwards, the MTT solution was removed and formed formazan crystals were dissolved with $100 \mu \mathrm{L}$ of DMSO. After several times aspiration, the absorbance was measured at $570 \mathrm{~nm}$ using a Microplate Reader (Model 680 S/N 21116).

\section{Induction of neural differentiation}

To induce neural differentiation, WJMSCs were cultured at $4 * 104$ per well in a 48 -well plate (SPL, China), including RA-loaded CH-hydrogel. After $24 \mathrm{~h}$ culture medium was replaced with differentiation medium containing (Zhang et al., 2006): DMEM/F-12 (DMEM/F12) (Gibco), 10\% KnockOut serum replacement (KSR) (Gibco), $20 \mathrm{ng} / \mathrm{mL}$ epidermal growth factor (EGF) (Invitrogen), $20 \mathrm{ng} / \mathrm{mL}$ basic fibroblast growth factor (bFGF) (Invitrogen) and $10 \mathrm{mg} / \mathrm{mL}$ heparin (Invitrogen). After 4 days, the cell's morphology was evaluated by Phase Contrast Microscope. Also, real-time-polymerase chain reaction (PCR) was performed to evaluate neural differentiation in treated WJMSCs.

Ribonucleic acid (RNA) extraction and real-time polymerase chain reaction (PCR)

Ribonucleic acid (RNA) extraction was performed using the Qiagen kit according to manufacturer protocols. The concentration of RNA in each sample was measured by the nanodrop (Thermofisher, USA). Afterward, total RNA was converted to complementary DNA (cDNA) using an RT2 HT First Strand Kit (Qiagen, Germany) according to the manufacturer's protocol. Finally, for SYBR green-based real-time PCR, $10 \mu \mathrm{L}$ SYBR green, $1 \mu \mathrm{L}$ Rox, $0.7 \mu \mathrm{L}$ F primer, $0.7 \mu \mathrm{L}$ R primer, $1 \mu \mathrm{L}$ complementary DNA (cDNA), and $6.4 \mu \mathrm{L}$ RNase free water were mixed in $20 \mu \mathrm{L}$ total volume and PCR performed in triplicate using ABI Step One device (Applied Biosystems, Sequences Detection Systems, Foster City, CA) for 40 cycles. (Table 1). The target gene expression level (nestin and $\beta$-tubulin III) was normalized to the reference gene ( $\beta$-actin) using the $\Delta \Delta \mathrm{CT}$ formula.

\section{Statistical analysis}

Data were expressed as Mean \pm SD of three separate experiments. One-way analysis of variance (ANOVA) was performed using SPSS software, version 23. Statistical significance was set at $\mathrm{P}<0.05$.

\section{Results}

\section{WJMSCs isolation and characterization:}

Seven days after isolation, most of the attached cells were bipolar and spindle-shaped (Figure 2A). After 3 passages, cells expressed CD44, CD105, CD90, and CD73 (98.6\%, 91.4\%, 98.3\%, and 95.9\%, respectively) and most of the cells expressed CD45 and CD34 (4.75\%, and $2.74 \%$, respectively) were negative as hematopoietic markers (Figure 1). 


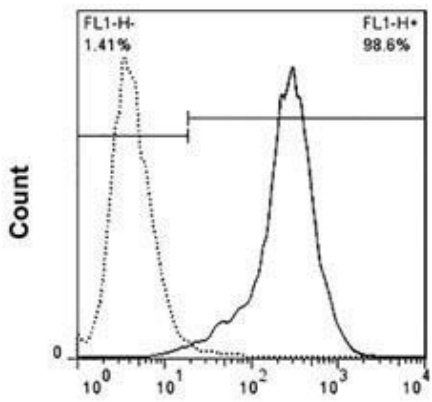

A) CD44-FITC

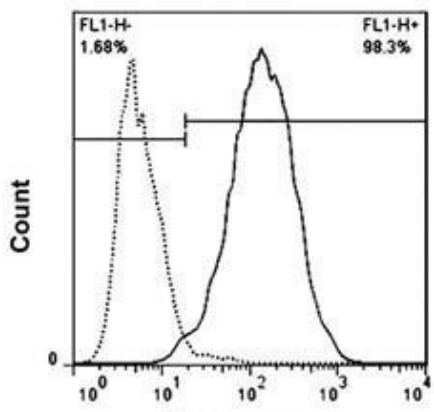

C)CD90-FITC

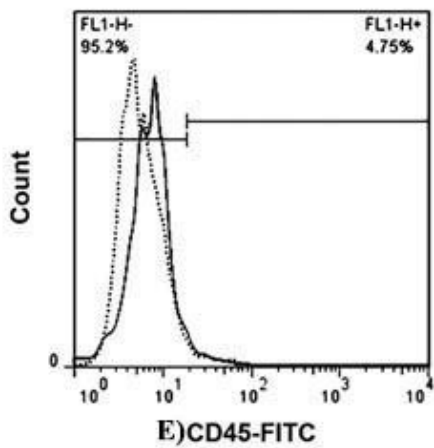

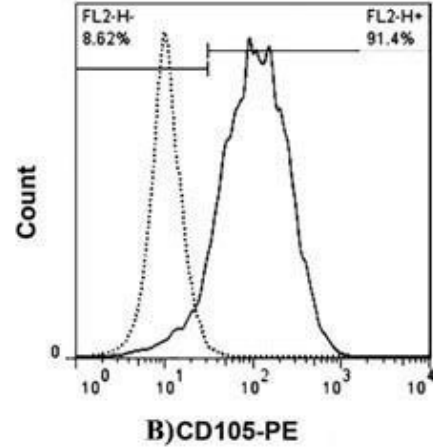
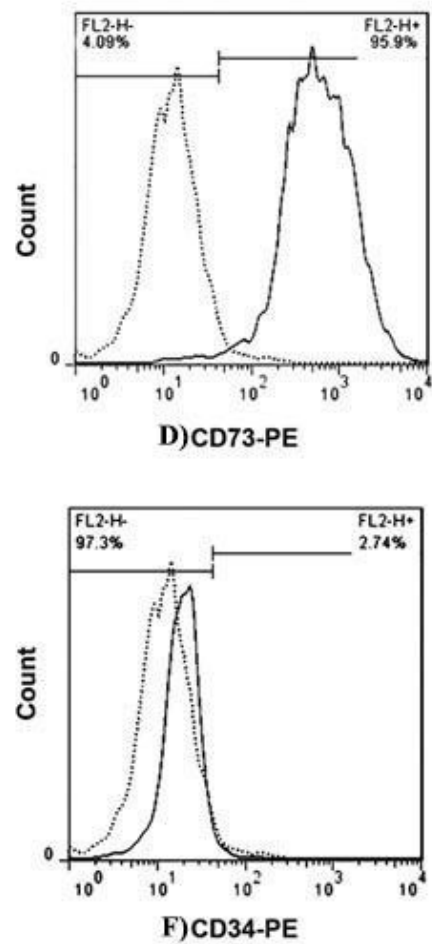

NEUROSCIENCE

Figure 1. Flow cytometry analysis of wharton's jelly-derived mesenchymal stem cells (WJMSCs) showing positive marker

A.CD44: 98.60\%; B.CD105: 91.40\%; C.CD90: 98.30\%; D.CD73: 95.90\% and negative marker; E.CD45: 4.75\%; F.CD34: $2.74 \%$.

Hydrogel characterization

SEM images showed a highly-connected porous structure in CH-hydrogel (Figure 3). The results of determining gelling time showed that immediately after transferring the hydrogel to the oven (constant temperature of $37^{\circ} \mathrm{C}$ ), the hydrogel began to gel and became gelled completely after about 25 minutes.

Two, five diphenyl tetrazolium bromide (MTT) assay

MTT assay analysis showed no significant difference between the test and control groups $(\mathrm{P}<0.05)$. Results showed that using hydrogel had a direct effect on increasing the percentage of cell viability (Figure 4). During this period, the control group has a decrease in cell viability, while in other groups, it increased. The effect of the presence of RA is obvious in Figure 4.

\section{Gene expression analysis}

RT-PCR analysis demonstrated that Nestin and $\beta$-Tubulin III gene expression increased significantly in $\mathrm{RA}, \mathrm{CH}$-hydrogel, and RA-loaded $\mathrm{CH}$-hydrogel groups without any additional treatment compared with the control group. Furthermore, nestin and $\beta$-tubulin III expression levels were higher in groups that were treated with 
Table 1. Sequence of forward and reverse primers

\begin{tabular}{cl}
\hline Gene & Forward and Reverse Sequence \\
\hline$\beta$-actin & $F:\left(5^{\prime}-3^{\prime}\right):$ acatcaaggagaagctgtgctac \\
& R: $\left(3^{\prime}-5^{\prime}\right):$ cttcatgatggagttgaaggtagtt \\
Nestin & $F:\left(5^{\prime}-3^{\prime}\right):$ ggcttctctcagcatcttgg \\
R: $\left(3^{\prime}-5^{\prime}\right):$ aaggctggcataggtgtgtc \\
$\beta$-tubulin III & F: $\left(5^{\prime}-3^{\prime}\right):$ cagagcaagaacagcagctactt \\
& R: $\left(3^{\prime}-5^{\prime}\right):$ gtgaactccatctcgtccatgccctc \\
\hline
\end{tabular}

NEUR:SCIENCE

a neural differentiation medium compared to groups that did not treat with a differentiation medium. Results also showed that the highest expression level of nestin and $\beta$-tubulin III was detected in WJMSCs that were seeded on RA-loaded $\mathrm{CH}$-hydrogel in the presence of neural differentiation medium (Figure 5). The results also show that treatment of cells with rosmarinic acid in two-dimensional conditions also increases the expression of neural markers. However, cell culture in three-dimensional conditions is more crucial than treatment with rosmarinic acid alone $\left({ }^{*} \mathrm{P}<0.05,{ }^{* *} \mathrm{P}<0.01,{ }^{* * *} \mathrm{P}<0.005,{ }^{* * * *} \mathrm{P}<0.001\right)$.

\section{Discussion}

Our results showed that cells isolated from human Wharton jelly (by the method used in this study) express mesenchymal markers well and do not express CD34 hematopoietic marker and CD45 as a leukocyte marker. WJMSCs have a higher proliferation potential than BMSCs. The process of senescence in WJMSCs also occurs later than BMSCs (Batsali et al., 2013). Besides the advantages mentioned, human Wharton's jelly-derived mesenchymal stem cells (hWJMSCs) have enhanced expression of neurotrophic factors, and a spontaneous tendency toward a neural lineage differentiation compared to MSCs isolated from adult tissues (Millán-Rivero et al., 2018). The advantages of WJMSCs have led to many studies focusing on the differentiation of these cells into neuronal cells. In a study, hWJMSCs were neutrally differentiated and expressed specified neural markers at both the protein and mRNA levels, confirming that it is possible to generate neural stem cell-like cells from
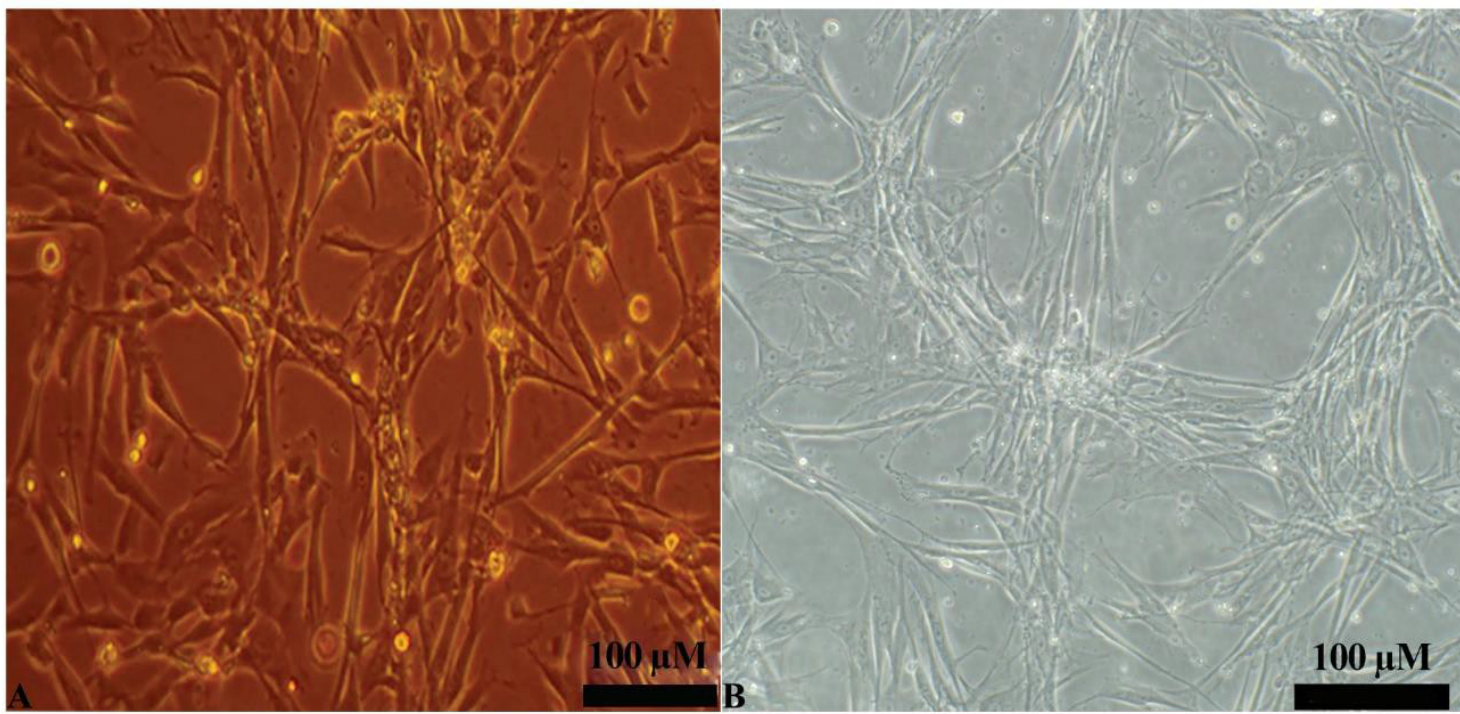

NEUR:SCIENCE

Figure 2. The inverted microscope image of WJMSCs before and after treatment with neural differentiation medium A) WJMSC) before any treatment in culture medium, B) Four days after treatment with neural differentiation medium 


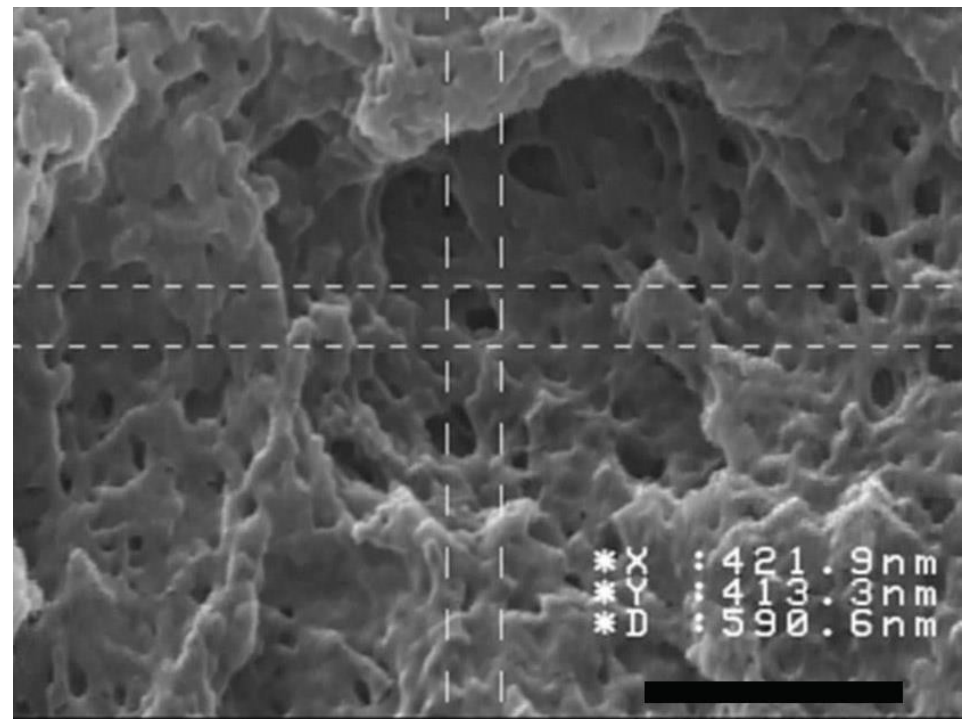

NEUR SCIENCE

Figure 3. The scanning electron microscopy (SEM) image of chitosan, beta-glycerol phosphate, and hydroxyl ethyl cellulose (CH- $\beta$-GP-HEC) hydrogel after drying

The right side shows the average pore size, which is less than $1 \mu \mathrm{m}$. Scale bar, $2 \mu \mathrm{M}$.

hWJMSCs in a 2D culture (Kruminis-Kaszkiel et al., 2020). Cell-based tissue engineering is a promising way to treat neurodegenerative diseases, such as Parkinson's disease (Alizadeh et al., 2019). The neuroprotective effects of rosmarinic acid have been investigated in various studies (Fazel Nabavi et al., 2015; Taram et al., 2018). In a study, Hwang and colleagues showed that rosmarinic acid has neuroprotective effects via reducing cholinergic activity and has a positive effect on neural plasticity (Hwang et al., 2016). Another research has shown that the treatment of cells with RA prevents $\mathrm{H}_{2} \mathrm{O}_{2}$-induced cell death in N2A cells (Ghaffari et al., 2014). Peng et al. demonstrated that treating WJMSC with a cocktail of growth factors leads to differentiation into Schwann-cell phenotype and promotes neurite outgrowth (Peng et al.,
2011). In another study, the effect of RA on spinal cord injury, significantly reduced the neurological deficit, increased neuronal preservation, and decreased apoptosis were observed. Ma et al. proved that RA's neuroprotective effect may be due to its antioxidant and anti-inflammatory properties (Ma et al., 2020).

The gelation time for hydrogels, including $\mathrm{CH}$-hydrogel, can range from a few minutes to several hours. The optimal gelation time for clinical applications is approximately 20 minutes. Our results showed that the gelatin time of the CH- $\beta$ GP-HEC compound was about 25 minutes. In many studies, the combination of chitosan and glycerol phosphate has been used to produce thermalsensitive hydrogels. MTT assay showed that $\mathrm{CH}$-hydro-

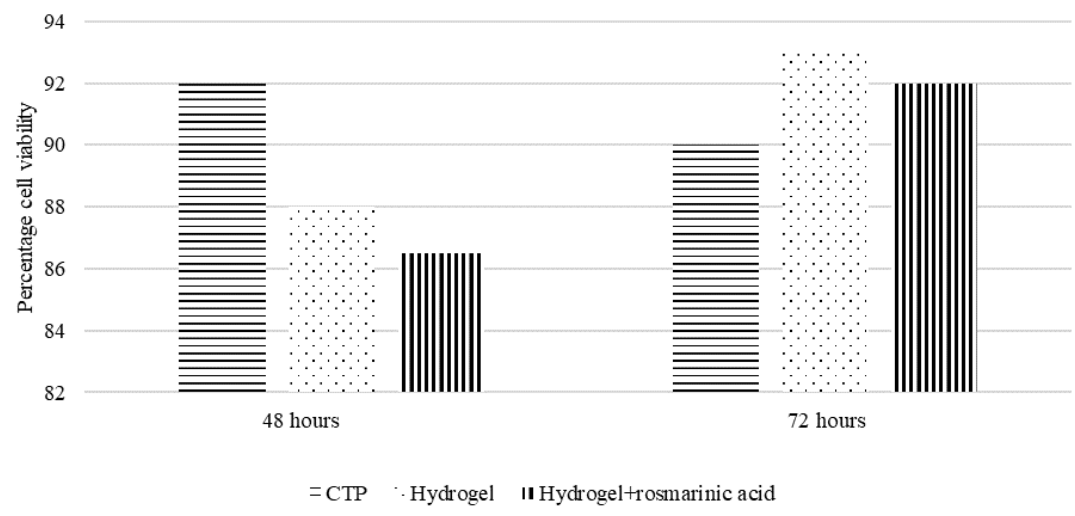

Figure 4. The results of MTT Assay

It shows that treatment of WJMSCs with rosmarinic acid leads to and incretion of cell viability. 


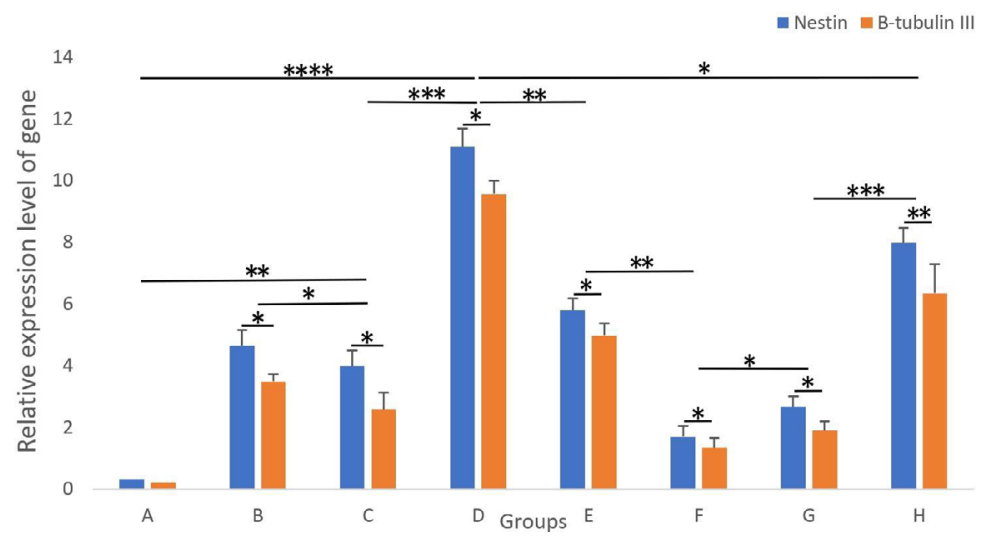

NEUR SCIENCE

Figure 5. Relative Gene expression level of nestin and $\beta$-tubulin III gene 4 days after treatment
A) WJSCs1+DMEM/F12
B) WJMSCs+neural differentiation medium
C) WJMSCs+Chitosan, beta-glycerol phosphate \&CH- $\beta$-GP-HEc hydrogel loaded with RA+DMEM/F12
D) WJMSCs+Chitosan, beta-glycerol phosphate \& $\mathrm{CH}-\beta-\mathrm{GP}-\mathrm{HEC}$ hydrogel loaded with RA+neural differentiation medium
E) WJMSCs+RA+neural differentiation medium
F) WJMSCs+RA+ DMEM/F12

G)WJMSCs+Chitosan, beta-glycerol phosphate \& HCH- $\beta$-GP-HEC hydrogel+DMEM/F12, H) WJMSCs+chitosan, beta-glycerol phosphate \& $\mathrm{CH}-\beta$-GP-HEC hydrogel+neural differentiation medium

The gene expression results show that the highest expression is observed in cells cultured in 3D hydrogel containing rosmarinic acid and differentiation medium. The results also show that treatment of cells with rosmarinic acid in 2D conditions also increases the expression of neural markers. However, cell culture in $3 \mathrm{D}$ conditions is more critical than treatment with rosmarinic acid alone. $\left({ }^{*} \mathrm{P}<0.05,{ }^{* *} \mathrm{P}<0.01,{ }^{* * *} \mathrm{P}<0.005,{ }^{* * * *} \mathrm{P}<0.001\right)$

gel had no cytotoxic effects on cultured WJMSCs. The results of cytotoxicity tests showed that the concentration of glycerol phosphate (GP) used in this study have been appropriate for causing osmolarity and appropriate $\mathrm{pH}$ in chitosan. Chitosan amine groups were also well neutralized with glycerol phosphate (GP), which aids in the gelation process of the compound by HEC at body temperature. Similar concentrations have been used in other papers to produce $\mathrm{CH}-\beta \mathrm{GP}-\mathrm{HEC}$ hydrogels, and similar results have been obtained (Naderi-Meshkin et al., 2014). The morphology of CH- $\beta$ GP-HEC hydrogels was observed by SEM (Figure 3). According to Fig. 3, the porous of the lyophilized hydrogels are homogeneous and have interconnected structures. This structure is suitable for maintaining cells and other compounds. For cell culture or tissue engineering applications, the number and size of hydrogels' pores are essential to transfer sufficient oxygen, eliminate toxic constituents, and provide sufficient space for cell growth (Sung et al., 2013). The pore and size of pores determine the type of cell to be cultured and the hydrogel's specific application (Bodenberger et al., 2016). Gene expression analysis showed that nestin and $\beta$-tubulin III expression as markers of neuronal differentiation increased in all groups compared to the control group, which included WJMSCs cultured in 2D conditions. Nestin is a neural stem cell marker and the tubulin marker is an early stage of neuronal differentiation. The highest expression of neuronal markers was observed in group $\mathrm{D}$, which included WJMSCs cultured on RA-loaded CH- $\beta$ GP-HEC hydrogel and treated with a neural differentiation medium. The results also show that RA alone can induce the expression of neural markers, although the expression level of neural markers in the RA group is significantly lower than in a group with a differentiation medium. Numerous studies have emphasized the protective effect of rosmarinic acid on neural cells (Cui et al., 2018; Ghaffari et al., 2014; Taram et al., 2018). Ferdousi et al. showed that using RA as a natural inducer for stem cells is a great advantage for cell-based therapies with good 
gene expression to neural differentiation (Ferdousi et al., 2019). It has also been shown that RA and its derivatives have positive effects on the proliferation of neural stem cell (NSC), neural stem cell (NSC) differentiation into neural precursor cells, as well as the maturation of neural precursor cells (Habtemariam, 2018). The results also revealed that the expression of neural markers in the $3 \mathrm{D}$ groups was significantly higher than in the $2 \mathrm{D}$ groups. As can be seen from the results, three-dimensional cell culture in hydrogels alone and without any additional factor can induce neural markers' expression. In addition, the effect of $3 \mathrm{D}$ culture on the expression of neural markers is more than that of rosmarinic acid. The impact of 3D culture on the induction of neural differentiation has also been shown in other studies (Chandrasekaran et al., 2017; Marchini et al., 2019; Song et al., 2018). Khodabandeh et al. used 2D collagen films and 3D collagen scaffolds for the culture of hWJMSCs. The cells showed a significant increase when cultured in 3D one compared to conventional monolayer culture and 2D films. They observed a significant increase in tight junction markers, such as claudin (Khodabandeh et al., 2016). Additionally, WJMSCs were differentiated into neurons in 3D alginate scaffolds and 2D monolayer systems. Hosseini et al realized that neural differentiation of embryonic stem cells was also more obviously increased in $3 \mathrm{D}$ culture compared to the $2 \mathrm{D}$ system. Furthermore, cells on alginate scaffolds exhibited a round appearance, which confirms the scaffold's suitability for culturing (Hosseini et al., 2015). Given the increased expression of the nestin gene compared to tubulin, 3D culture and rosmarinic acid seem to differentiate WJMSCs into neural stem cells. As a result, additional treatments may also be needed to differentiate stem cells into mature neurons.

\section{Conclusion}

In addition to its anti-inflammatory role, rosmarinic acid can be used as a facilitator of neuronal differentiation. The results of this study showed that although rosmarinic acid alone can induce the expression of neural markers if used in combination with an inducer of neural differentiation, it will have a greater effect on the expression of neural markers. On the other hand, stem cell culture in three-dimensional conditions with rosmarinic acid helps to increase the expression of neural markers. The results of this study and such studies could lead to the production of inexpensive 3D scaffolds capable of successfully inducing neural differentiation in stem cells, which can be used in nerve tissue engineering.

\section{Ethical Considerations}

\section{Compliance with ethical guidelines}

All tests performed in this study was done under approval of Ethical Committee of Najafabad Branch, Islamic Azad University.

\section{Funding}

This study was part of Mohsen Salmanvandi thesis, Department of Material Engineering, Najafabad Branch, Islamic Azad University.

\section{Authors' contributions}

All authors equally contributed to preparing this article.

\section{Conflict of interest}

The authors declared no conflict of interest.

\section{Acknowledgments}

We would like to thank all colleagues in Cellular and Molecular Research Center of Iran University of Medical Sciences.

\section{References}

Adomako-Bonsu, A. G., Chan, S. L., Pratten, M., \& Fry, J. R (2017). Antioxidant activity of rosmarinic acid and its principal metabolites in chemical and cellular systems: Importance of physico-chemical characteristics. Toxicology in Vitro: An International Journal Published in Association with BIBRA, 40, 248-255. [DOI:10.1016/j.tiv.2017.01.016] [PMID]

Alizadeh, R., Bagher, Z., Kamrava, S. K., Falah, M., Ghasemi Hamidabadi, H., \& Eskandarian Boroujeni, M., et al. (2019). Differentiation of human mesenchymal stem cells (MSC) to dopaminergic neurons: A comparison between Wharton's Jelly and olfactory mucosa as sources of MSCs. Journal of Chemical Neuroanatomy, 96, 126-133. [DOI:10.1016/j. jchemneu.2019.01.003] [PMID]

Allahbakhshi, E., Hashemitabar, M., Shariati, M., Tabandeh, M R., \& Solgi, G. (2013). Differentiation of the definitive endoderm from Wharton's jelly mesenchymal stem cells (WJMSC). Journal of Biological Research, 20, 217-227. [Link]

Bagher, Z., Azami, M., Ebrahimi-Barough, S., Mirzadeh, H., Solouk, A., \& Soleimani, M., et al. (2016). Differentiation of Wharton's jelly-derived mesenchymal stem cells into motor neuron-like cells on three-dimensional collagen- grafted nanofibers. Molecular Neurobiology, 53(4), 2397-2408. [DOI:10.1007/s12035-015-9199-x] [PMID] 
Batsali, A., Pontikoglou, C., Kouvidi, E., Damianaki, A., Stratigi, A., \& Kastrinaki, M. C., et al. (2013). Comparative analysis of bone marrow and wharton's jelly mesenchymal stem/ stromal cells. Blood, 122(21), 1212. [DOI:10.1182/blood. V122.21.1212.1212]

Bharti, D., Shivakumar, S. B., Park, J. K., Ullah, I., Subbarao, R. B., \& Park, J. S., et al. (2018). Comparative analysis of human Wharton's jelly mesenchymal stem cells derived from different parts of the same umbilical cord. Cell and Tissue Research 372, 51-65. [DOI:10.1007/s00441-017-2699-4]

Bodenberger, N., Kubiczek, D., Abrosimova, I., Scharm, A., Kipper, F., \& Walther, P., et al. (2016). Evaluation of methods for pore generation and their influence on physio-chemical properties of a protein based hydrogel. Biotechnology Reports, 12, 6-12. [DOI:10.1016/j.btre.2016.09.001] [PMID] [PMCID]

Chandrasekaran, A., Avci, H. X., Ochalek, A., Rösingh, L. N., Molnár, K., \& László, L., et al. (2017). Comparison of 2D and 3D neural induction methods for the generation of neural progenitor cells from human induced pluripotent stem cells. Stem Cell Research, 25, 139-151. [DOI:10.1016/j.scr.2017.10.010]

Crompton, K. E., Goud, J. D., Bellamkonda, R. V., Gengenbach, T. R., Finkelstein, D. I., \& Horne, M. K., et al. (2007) Polylysine-functionalised thermoresponsive chitosan hydrogel for neural tissue engineering. Biomaterials, 28(3), 441-449. [DOI:10.1016/j.biomaterials.2006.08.044] [PMID]

Cui, H. Y., Zhang, X. J., Yang, Y., Zhang, C., Zhu, C. H., \& Miao, J. Y., et al. (2018). Rosmarinic acid elicits neuroprotection in ischemic stroke via Nrf2 and heme oxygenase 1 signaling. Neural Regeneration Research, 13(12), 2119-2128. [DOI:10.4103/1673-5374.241463] [PMID] [PMCID]

Nabavi, S. F., Tenore, G. C., Daglia, M., Tundis, R., Loizzo, M. R., \& Nabavi, S. M. (2015). The cellular protective effects of rosmarinic acid: From bench to bedside. Current Neurovascular Research, 12(1), 98-105. [DOI:10.2174/156720261266615010 9113638] [PMID]

Ferdousi, F., Sasaki, K., Uchida, Y., Ohkohchi, N., Zheng, Y. W., \& Isoda, H. (2019). Exploring the potential role of rosmarinic acid in neuronal differentiation of human amnion epithelial cells by microarray gene expression profiling. Frontiers in Neuroscience, 13, 779. [DOI:10.3389/fnins.2019.00779] [PMID] [PMCID]

Fonteles, A. A., de Souza, C. M., de Sousa Neves, J. C., Menezes, A. P., Santos do Carmo, M. R., \& Fernandes, F. D., et al. (2016). Rosmarinic acid prevents against memory deficits in ischemic mice. Behavioural Brain Research, 297, 91-103. [PMID]

Fregnan, F., Ciglieri, E., Tos, P., Crosio, A., Ciardelli, G., \& Ruini, F., et al. (2016). Chitosan crosslinked flat scaffolds for peripheral nerve regeneration. Biomedical Materials (Bristol, England), 11(4), 045010. [PMID]

Ghaffari, H., Venkataramana, M., Jalali Ghassam, B., Chandra Nayaka, S., Nataraju, A., \& Geetha, N. P., et al. (2014). Rosmarinic acid mediated neuroprotective effects against $\mathrm{H}_{2} \mathrm{O} 2-$ induced neuronal cell damage in N2A cells. Life Sciences, 113(1-2), 7-13. [DOI:10.1016/j.lfs.2014.07.010] [PMID]

Gnavi, S., Barwig, C., Freier, T., Haastert-Talini, K., Grothe, C., \& Geuna, S. (2013). The use of chitosan-based scaffolds to enhance regeneration in the nervous system. International Review of Neurobiology, 109, 1-62. [PMID]
Habtemariam, S. (2017). Antidiabetic potential of monoterpenes A case of small molecules punching above their weight. International Journal of Molecular Sciences, 19(1), 4. [PMID] [PMCID]

Haddad-Mashadrizeh, A., Matin, M. M., Bahrami, A. R., Edalatmanesh, M. A., Naderi- Meshkin, H., \& Mousavi, S., et al. (2013). Cytotoxicity and biocompatibility evaluation of chitosan-beta glycerol phosphate-hydroxyethyl cellulose hydrogel on adult rat liver for cell-based therapeutic applications. International Journal of Biomedical Engineering and Technology, 12(3), 228-239. [DOI:10.1504/IJBET.2013.057260]

Hamaguchi, T., Ono, K., Murase, A., \& Yamada, M. (2009). Phenolic compounds prevent Alzheimer's pathology through different effects on the amyloid- $\beta$ aggregation pathway. The American Journal of Pathology, 175(6), 2557-2565. [DOI:10.2353/ ajpath.2009.090417] [PMID] [PMCID]

Hase, T., Shishido, S., Yamamoto, S., Yamashita, R., Nukima H., \& Taira, S., et al. (2019). Rosmarinic acid suppresses Alzheimer's disease development by reducing amyloid $\beta$ aggregation by increasing monoamine secretion. Scientific Reports, 9(1), 8711. [DOI:10.1038/s41598-019-45168-1] [PMID] [PMCID]

Hosseini, S. M., Vasaghi, A., Nakhlparvar, N., Roshanravan R., Talaei-Khozani, T., \& Razi, Z. (2015). Differentiation of Wharton's jelly mesenchymal stem cells into neurons in alginate scaffold. Neural Regeneration Research, 10(8), 1312-1316. [DOI:10.4103/1673-5374.162768] [PMID] [PMCID]

Hwang, E. S., Kim, H. B., Choi, G. Y., Lee, S., Lee, S. O., \& Kim, S et al. (2016). Acute rosmarinic acid treatment enhances longterm potentiation, BDNF and GluR-2 protein expression, and cell survival rate against scopolamine challenge in rat organotypic hippocampal slice cultures. Biochemical and Biophysical Research Communications, 475(1), 44-50. [DOI:10.1016/j. bbrc.2016.04.153] [PMID]

Jamalpoor, Z., Taromi, N., Soleimani, M., Koudehi, M. F., \& Asgari, A. (2019). In vitro interaction of human Wharton's jelly mesenchymal stem cells with biomimetic 3D scaffold. Journal of Biomedical Materials Research Part A, 107(6), 1166-1175. [DOI:10.1002/jbm.a.36608] [PMID]

Karimpour Malekshah, A., Talebpour Amiri, F., Ghaffari, E., Alizadeh, A., Jamalpoor, Z., \& Mirhosseini, M., et al. (2016) Growth and chondrogenic differentiation of mesenchymal stem cells derived from human adipose tissue on chitosan scaffolds. Journal of Babol University of Medical Sciences, 18(9), 32-38. [Link]

Khodabandeh, Z., Vojdani, Z., Talaei-Khozani, T., Jaberipour, M., Hosseini, A., \& Bahmanpour, S. (2016). Comparison of the expression of hepatic genes by human wharton's jelly mesenchymal stem cells cultured in 2D and 3D collagen culture systems. Iranian Journal of Medical Sciences, 41(1), 28-36. [Link]

Kruminis-Kaszkiel, E., Osowski, A., Bejer-Oleńska, E., Dziekoński, M., \& Wojtkiewicz, J. (2020). Differentiation of human mesenchymal stem cells from wharton's jelly towards neural stem cells using a feasible and repeatable protocol Cells, 9(3), 739. [DOI:10.3390/cells9030739] [PMID] [PMCID]

Liu, L., Gao, Q., Lu, X., \& Zhou, H. (2016). In situ forming hydrogels based on chitosan for drug delivery and tissue regeneration. Asian Journal of Pharmaceutical Sciences, 11(6), 673-683. [DOI:10.1016/j.ajps.2016.07.001] 
Lu, B., Nagappan, G., \& Lu, Y. (2015). BDNF and synaptic plasticity, cognitive function, and dysfunction. Handbook of Experimental Pharmacology, 220, 223-250. [DOI:10.1007/978-3-64245106-5_9] [PMID]

Ma, Z., Lu, Y., Yang, F., Li, S., He, X., \& Gao, Y., et al. (2020). Rosmarinic acid exerts a neuroprotective effect on spinal cord injury by suppressing oxidative stress and inflammation via modulating the Nrf2/HO-1 and TLR4/NF-kB pathways. Toxicology and Applied Pharmacology,397, 115014. [PMID]

Marchini, A., Raspa, A., Pugliese, R., El Malek, M. A., Pastori, V., \& Lecchi, M., et al. (2019). Multifunctionalized hydrogels foster hNSC maturation in 3D cultures and neural regeneration in spinal cord injuries. Proceedings of the National Academy of Sciences of the United States of America, 116(15), 7483-7492. [DOI:10.1073/pnas.1818392116] [PMID] [PMCID]

Millán-Rivero, J. E., Nadal-Nicolás, F. M., García-Bernal, D. Sobrado-Calvo, P., Blanquer, M., \& Moraleda, J. M., et al. (2018). Human Wharton's jelly mesenchymal stem cells protect axotomized rat retinal ganglion cells via secretion of antiinflammatory and neurotrophic factors. Scientific Reports, 8 , 16299. [DOI:10.1038/s41598-018-34527-z] [PMID] [PMCID]

Naderi-Meshkin, H., Andreas, K., Matin, M. M., Sittinger, M., Bidkhori, H. R., \& Ahmadiankia, N., et al. (2014). Chitosanbased injectable hydrogel as a promising in situ forming scaffold for cartilage tissue engineering. Cell Biology International, 38(1), 72-84. [DOI:10.1002/cbin.10181]

Nieto, G., Ros, G., \& Castillo, J. (2018). Antioxidant and antimicrobial properties of rosemary (rosmarinus officinalis, L.): A review. Medicines, 5(3), 98. [DOI:10.3390/medicines5030098] [PMCID]

Pankongadisak, P., \& Suwantong, O. (2018). The potential use of thermosensitive chitosan/silk sericin hydrogels loaded with longan seed extract for bone tissue engineering. RSC Advances, 8(70), 40219-40231. [DOI:10.1039/C8RA07255H] [PMID] [PMCID]

Peng, J., Wang, Y., Zhang, L., Zhao, B., Zhao, Z., \& Chen, J. F., et al. (2011). Human umbilical cord Wharton's jelly-derived mesenchymal stem cells differentiate into a Schwann-cell phenotype and promote neurite outgrowth in vitro. Brain Research Bulletin, 84(3), 235-243. [DOI:10.1016/j.brainresbull.2010.12.013] [PMID]

Rust, R., \& Kaiser, J. (2017). Insights into the dual role of inflammation after spinal cord injury. The Journal of Neuroscience: The Official Journal of the Society for Neuroscience, 37(18), 4658-4660. [DOI:10.1523/JNEUROSCI.0498-17.2017] [PMID] [PMCID]

Schneider, R. K., Puellen, A., Kramann, R., Raupach, K., Bornemann, J., \& Knuechel, R., et al. (2010). The osteogenic differentiation of adult bone marrow and perinatal umbilical mesenchymal stem cells and matrix remodelling in threedimensional collagen scaffolds. Biomaterials, 31(3), 467-480. [DOI:10.1016/j.biomaterials.2009.09.059] [PMID]

Shen, X., Shamshina, J. L., Berton, P., Gurau, G., \& Rogers, R. D. (2015). Hydrogels based on cellulose and chitin: Fabrication, properties, and applications. Green Chemistry, 18(1), 53-75. [DOI:10.1039/c5gc02396c]

Simorgh, S., Alizadeh, R., Eftekharzadeh, M., Haramshahi, S. M A., Milan, P. B., \& Doshmanziari, M., et al. (2019). Olfactory mucosa stem cells: An available candidate for the treatment of the Parkinson's disease. Journal of Cellular Physiology, 234(12), 23763-23773. [DOI:10.1002/jcp.28944] [PMID]
Song, L., Tsai, A. C., Yuan, X., Bejoy, J., Sart, S., \& Ma, T., et al. (2018). Neural Differentiation of Spheroids Derived from Human Induced Pluripotent Stem Cells- Mesenchymal Stem Cells Coculture. Tissue Engineering Part A, 24(11-12), 915-929. [DOI:10.1089/ten.tea.2017.0403] [PMID] [PMCID]

Sung, K. E., Su, X., Berthier, E., Pehlke, C., Friedl, A., \& Beebe, D. J. (2013). Understanding the impact of $2 \mathrm{D}$ and 3D fibroblast cultures on in vitro breast cancer models. PloS One 8(10), e76373. [DOI:10.1371/journal.pone.0076373] [PMID] [PMCID]

Supper, S., Anton, N., Seidel, N., Riemenschnitter, M., Curdy, C., \& Vandamme, T. (2014). Thermosensitive chitosan/glycerophosphate-based hydrogel and its derivatives in pharmaceutical and biomedical applications. Expert Opinion on Drug Delivery, 11(2), 249-267. [PMID]

Taram, F., Ignowski, E., Duval, N., \& Linseman, D. A. (2018). Neuroprotection comparison of rosmarinic acid and carnosic acid in primary cultures of cerebellar granule neurons. Mole cules, 23(11), 2956. [DOI:10.3390/molecules23112956] [PMID] [PMCID]

Valmikinathan, C. M., Mukhatyar, V. J., Jain, A., Karumbaiah, L., Dasari, M., \& Bellamkonda, R. V. (2012). Photocrosslinkable chitosan based hydrogels for neural tissue engineering. Soft Matter, 8(6), 1964-1976. [DOI:10.1039/c1sm06629c] [PMID] [PMCID]

Vawda, R., \& G. Fehlings, M. (2013). Mesenchymal cells in the treatment of spinal cord injury: Current \& future perspectives. Current Stem Cell Research \& Therapy, 8(1), 25-38. [DOI:1 0.2174/1574888x11308010005] [PMID]

Wang, H. S., Hung, S. C., Peng, S. T., Huang, C. C., Wei, H. M., \& Guo, Y. J., et al. (2004). Mesenchymal stem cells in the wharton's jelly of the human umbilical cord. Stem Cells, 22(7) 1330-1337. [DOI:10.1634/stemcells.2004-0013] [PMID]

Wang, J., Xu, H., Jiang, H., Du, X., Sun, P., \& Xie, J. (2012). Neurorescue effect of rosmarinic acid on 6-hydroxydopamine-lesioned nigral dopamine neurons in rat model of Parkinson's disease. Journal of Molecular Neuroscience, 47, pages113-119. [DOI:10.1007/s12031-011-9693-1]

Zhang, J. Q., Yu, X. B., Ma, B. F., Yu, W. H., Zhang, A. X., \& Huang, G., et al. (2006). Neural differentiation of embryonic stem cells induced by conditioned medium from neural stem cell. Neuroreport, 17(10), 981-986. [PMID]

Zhang, J., Lu, X., Feng, G., Gu, Z., Sun, Y., \& Bao, G., et al (2016). Chitosan scaffolds induce human dental pulp stem cells to neural differentiation: Potential roles for spinal cord injury therapy. Cell and Tissue Research, 366(1), 129-142. [DOI:10.1007/s00441-016-2402-1] [PMID]

Zulkifli, F. H., Hussain, F. S. J., Harun, W. S. W., \& Yusoff, M. M. (2019). Highly porous of hydroxyethyl cellulose biocomposite scaffolds for tissue engineering. International Journal of Biological Macromolecules, 122, 562-571. [DOI:10.1016/j.ijbiomac.2018.10.156] [PMID] 
This Page Intentionally Left Blank 